\title{
Bone metastases from head \& neck squamous cell carcinoma (HNSCC) - Reviewing the patients' background and imaging features mainly of whole body MRI (WBMRI)
}

\author{
Katsuyuki Nakanishi ${ }^{1}$, Mio Sakai ${ }^{1}$, Hiromitsu Sumikawa $^{1}$, Naoyuki Kanayama ${ }^{2}$, Kazuya Oshima ${ }^{3}$, Takashi Fujii ${ }^{4}$, Toshinari Yagi ${ }^{5}$, Shinichi \\ Nakatsuka ${ }^{6}$, Nobuo Kashiwagi ${ }^{7}$ and Noriyuki Tomiyama ${ }^{8}$ \\ ${ }^{1}$ Department of Diagnostic and Interventional Radiology, Osaka International Cancer Institute, 3-1-69, Otemae Chuo-ku, Osaka city, Osaka 541-8567, Japan \\ ${ }^{2}$ Department of Radiation Oncology, Osaka International Cancer Institute, 3-1-69, Otemae Chuo-ku, Osaka city, Osaka 541-8567, Japan \\ ${ }^{3}$ Department of Orthopaedic Surgery, Osaka International Cancer Institute, 3-1-69, Otemae Chuo-ku, Osaka city, Osaka 541-8567, Japan \\ ${ }^{4}$ Department of Otolaryngology Head and Neck Surgery, Osaka International Cancer Institute, 3-1-69, Otemae Chuo-ku, Osaka city, Osaka 541-8567, Japan \\ ${ }^{5}$ Department of Clinical Oncology, Osaka International Cancer Institute, 3-1-69, Otemae Chuo-ku, Osaka city, Osaka 541-8567, Japan \\ ${ }^{6}$ Department of Pathology, Osaka International Cancer Institute, 3-1-69, Otemae Chuo-ku, Osaka city, Osaka 541-8567, Japan \\ ${ }^{7}$ Department of Radiology, Kindai University Faculty of Medicine, 337-2, Ohno-higashi, Osakasayama city, Osaka 589-0014, Japan \\ ${ }^{8}$ Department of Diagnostic Radiology, Osaka University Graduate School of Medicine, 2 Yamadaoka, Suita-city Osaka 565-0871, Japan
}

\begin{abstract}
The aim of this article is to review images of bone metastases from Head \& Neck Squamous Cell Carcinoma (HNSCC) mainly of whole body MRI (WB-MRI). 14 cases of HNSCC were reviewed (2, nasopharynx; 3, oropharynx; 2, hypopharynx; 4, tongue; 2, larynx; 1 , nasal cavity). In four of these 14 cases, only bone metastases have been found more than 14 months. In all cases, the metastatic lesions were seen as high intensity on diffusion-weighted images (DWI) on MRI. In five of our 14 cases, osteosclerotic patterns were recognized in CT. In these five, two were nasopharyngeal carcinoma (NPC) and the other three were oropharyngeal carcinoma (OPC). In two cases of NPC, Epstein-Barr virus (EBV) and in three cases of OPC, Human papilloma virus (HPV) 16 were proven. In the other four cases, neither osteosclerotic nor lytic patterns were shown. Hence, they were classified as intertrabecular patterns. In the other five cases, osteolytic patterns were recognized along with the lesions. Bone metastases from HNSCC are rare but need to be detected. It is often found with lung or liver metastases, but it can appear alone. Although, on CT bone metastases could appear in various patterns, these lesions appear consistently as high intensity on DWI of MRI.
\end{abstract}

\section{Introduction}

Head and neck squamous cell carcinomas (HNSCC) are usually locally invasive, with a proclivity to metastasize to regional lymph nodes rather than to spread hematogenously [1-6]. Distant metastases usually occur late during the course of disease, most commonly in the lungs, followed by the bones and liver. Whole body MRI (WB-MRI) is now developing as a new non-invasive method, mainly for detecting bone metastases [2,7-9].

In this paper, we retrospectively reviewed the imaging features of bone metastases from HNSCC on WB-MRI mainly by reference to 18F-Fluorodeoxyglucose-positron emission tomography/CT (FDG$\mathrm{PET} / \mathrm{CT}$ ) and CT .

\section{Materials and methods}

\section{Patients' background}

We retrospectively reviewed the cases of WB-MRI for detecting bone metastases from 2013-2016. Fourteen cases, which were histologically proven HNSCCs of primary sites, were selected. Details of these 14 cases were summarized in Table 1 . All cases had a follow-up of at least six months after initial examination and possible metastatic sites not only bone but also other metastatic site such as lung and liver were confirmed radiologically and clinically adding either or both FDG-PET and contrast enhanced whole body CT. As far as we researched, other primary sites have not been found. All patients gave informed consent for undergoing WB-MRI. This retrospective study was approved by our institutional review board (IRB). Ethics Committee of Osaka International Cancer Institute approved this study in $13^{\text {th }}$ May 2016, as No. 160135027. This research on human subjects is in compliance with Helsinki Declaration [10].

In all patients, we reviewed the span of the detection of bone metastases, follow-up term and the existence of other metastases such as lung and liver.

Correspondence to: Katsuyuki Nakanishi MD, PhD, Department of Diagnostic and Interventional Radiology, Osaka International Cancer Institute, 3-1-69, Otemae Chuo-ku, Osaka city, Osaka 541-8567, Japan, Tel: 81669451181; Fax: 81669453329; E-mail: je2k-nkns@asahi-net.or.jp

Key words: head and neck squamous cell carcinoma (HNSCC), bone metastasis, intertrabecular metastases, whole body MRI, whole body DWI

Received: July 22, 2017; Accepted: August 21, 2017; Published: August 24, 2017 
Nakanishi K (2017) Bone metastases from head \& neck squamous cell carcinoma (HNSCC) - Reviewing the patients' background and imaging features mainly of whole body MRI (WBMRI)

\section{Image parameter of WB-MRI}

A 3 tesla MRI (Magnetom Trio 3T, Siemens, Erlangen, Germany) was utilized. The patients were covered with a 102 channel surface coil, positioned head first on an extended anatomical coverage table. Detailed imaging parameters were summarized in Table 2. Total spine sagittal images of STIR and T1WI (Turbo Spin Echo) (Table 2), coronal images of in phase and out of phase T1WI (Table 3), and axial images of DWI from lower neck to proximal femur (Table 4) were captured.

The time required by the patients to be immobile in the MRI bore was approximately 23 minutes. The total examination time, including the coil set up, patient positioning, image processing (MIP, MPR and Fusion) and the image layout for reading, was less than 60 minutes.

\section{Follow-up term and span of the detection of bone metastases}

The follow-up term from the initial detection ranged from six to 97 months and eight patients died due to their primary diseases (cases $4,6,7,8,9,11,12$ and 13 ). The span from the initial staging to the detection of bone metastases ranged from 0 to 42 months (average: 14.3 months).

\section{Onset of metastatic site}

In six of these 14 cases, bone metastases were independent newonset metastases (case 1,2,5,6,12 and 13). In four of these six (case $2,5,6,12$ ), only bone metastases have been found beyond 14 months.

In two cases (case 3 and 4), bone and liver metastases were together the new-onset metastases. Bone and lung metastases (case 8) and bone and adrenal metastases (case 7) were together the new-onset metastases.

In three cases (case 9,10 and 11), bone metastases were found secondary to lymph node metastases and in one case, they were found secondary to lung metastases (case 14).

\section{Distribution of bone metastases}

In 12 cases, multiple bone metastases were found on WB-MRI. In two cases, solitary metastasis was found (case 1 and 12).

\section{Image findings}

1) Osteosclerotic pattern ( $N=5)$ (case 1-5): In five cases, osteosclerotic patterns are recognized diffusely in CT (Figures1 and 2). In these five cases, these lesions are shown as high intensity on DWI.

2) Intertrabecular pattern $(\mathrm{N}=4)($ case 6,7,8 and 13): In four cases, CT showed neither osteosclerotic nor lytic changes which suggested intertrabecular pattern. In these cases, the multiple lesions were seen as high intensity on DWI and high accumulation on FDG-PET CT (Figure 3).

3) Osteolytic pattern $(\mathrm{N}=5)$ (case 9-12 and 14): In the other five cases, the lesions were shown as osteolytic pattern. In these cases, the multiple lesions were also shown as high intensity on DWI and high accumulation on FDG-PET CT (Figure 4).

\section{Discussion}

In the field of HNSCC, bone metastases have received less attention than pulmonary metastases in patients with HNSCC [1].

In our study group, in four (case 2,5,6,12), metastases were found in bone only. Kim, et al. found four patients of only bone metastases in 17 of bone metastases [1].

In our results, five cases of HNSCC were shown to be osteosclerotic (Case 1-5). In these five, two were nasopharyngeal carcinoma (NPC) and the other three were oropharyngeal carcinoma (OPC). Metastatic lesions of these five cases reveals as high intensity on DWI.
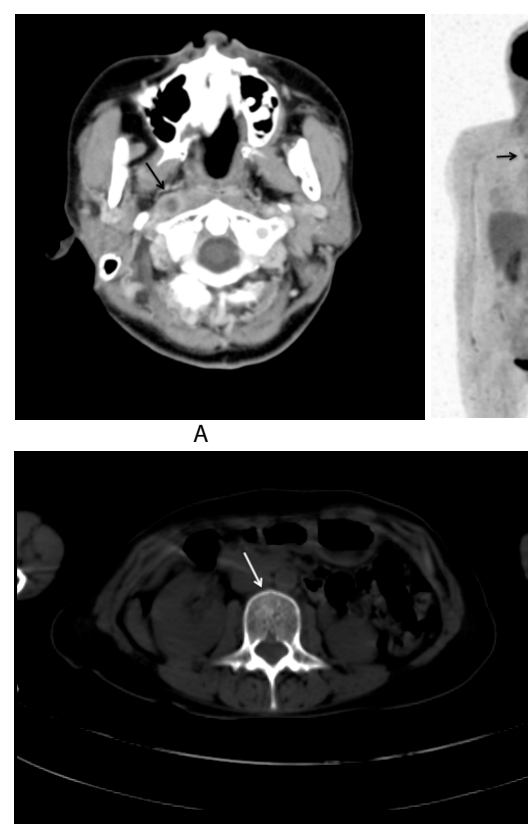

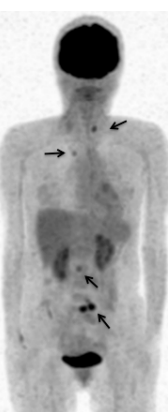

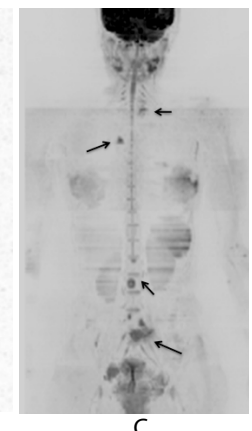

C

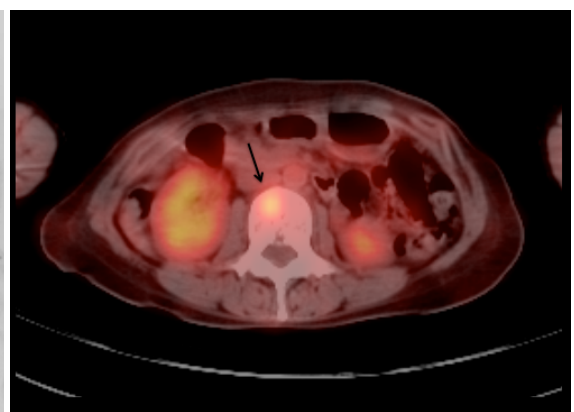

D

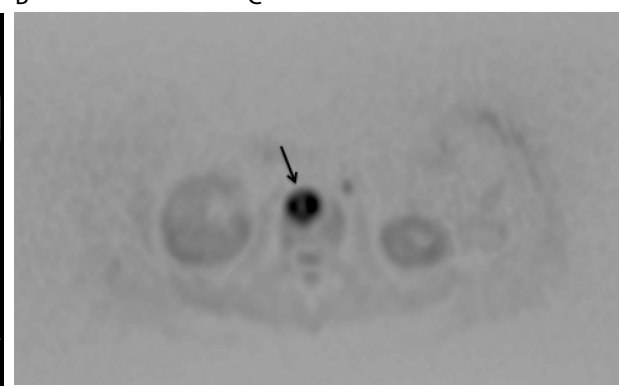

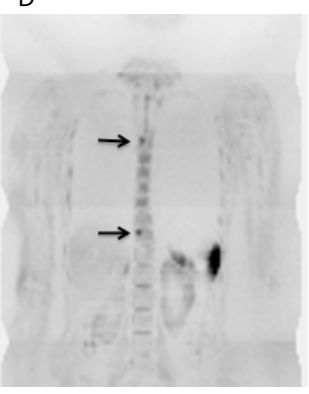

Figure 1. 42-year-old female with NPC T1N2M0 (Case 2) [A: On initial CT, right Ruby ale lymph node swelling is shown (arrow) and SCC was proved by right nasopharyngeal wall biopsy; B: On PET-MIP image 10 months after primary site detection, accumulated areas are shown in the vertebral bodies (arrows); C: On DWI-MIP image 36 days after FDG-PET (Fig.1B), high intensity areas are also shown in sacrum, L3 and Th5 and are well correlated with PET-MIP image (arrows); D: On PET-CT at the L3 level, same examination of Fig.1B, accumulation is seen (arrow); E: On CT at the L3 level, same examination of Fig 1B and D, subtle osteosclerotic change is seen (white arrow); F: On axial DWI at the L3 level, same examination of Fig.1C, high intensity area is seen (arrow). These areas are judged comprehensively as osteosclerotic bone metastases; G: On DWI coronal reconstructed image 27 months after primary site detection, new high intensity areas are shown in Th4 and Th10 (arrows). They are judged as new bone metastases.] This patient has been followed up 27 months and is confirmed to be alive. Only bone metastases are confirmed. 
Nakanishi K (2017) Bone metastases from head \& neck squamous cell carcinoma (HNSCC) - Reviewing the patients' background and imaging features mainly of whole body MRI (WBMRI)
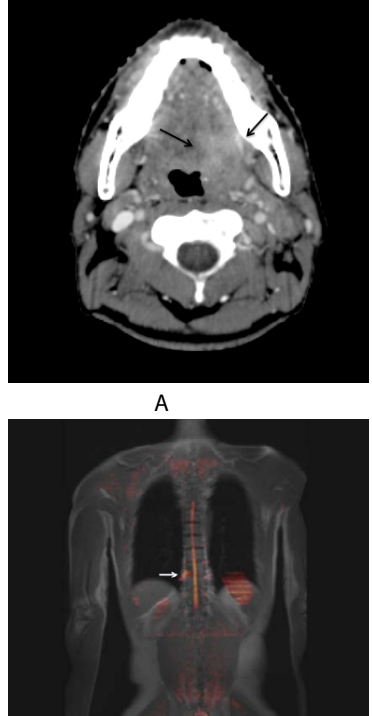

D

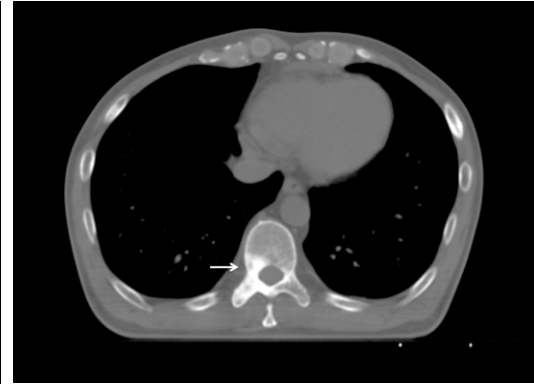

B

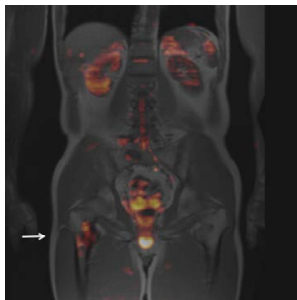

$\mathrm{E}$

$\mathrm{F}$
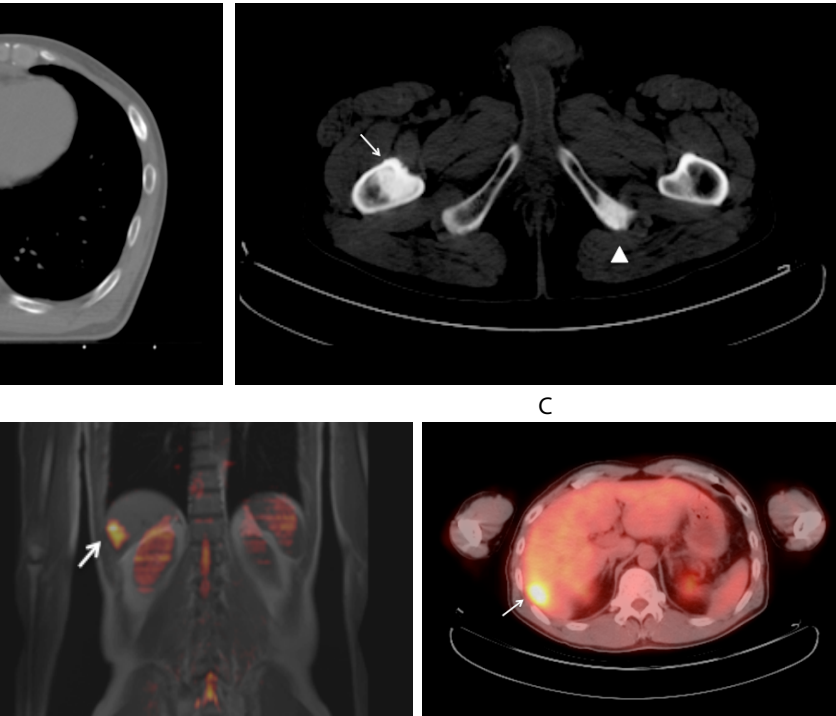

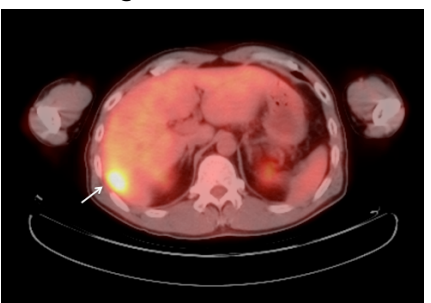

G

Figure 2. A 53-year-old male with OPC (Case 3). Its initial stage was T4aN2cM0 [A: On initial CT, mass is shown on the left oropharyngral wall (arrows) and SCC was proved by biopsy; B: On CT image 33 months after initial CT (Fig.2A), at the Th7 level, osteosclerotic pattern is shown (white arrow); C: On CT same examination of Fig.2B, at the right proximal femur level, osteosclerotic pattern is shown (white arrow). Also in the left ischium, osteosclerotic pattern is shown (white arrowhead); D: On WB-MRI examination 28 days after CT (Fig.2B and C), in coronal reconstructed fused T1WI and DWI, on the right pedicle of Th10, high intensity area is shown (white arrow) and diagnosed as bone metastasis; E: In same examination of Fig.2D, also on the right proximal femur, high intensity area is shown (white arrow) and diagnosed as bone metastasis. In both lesions, high accumulations are seen in PET-CT also and they are judged comprehensively as osteosclerotic metastases; F: In same WB-MRI examination of Fig.2D and E, high intensity area is shown in the liver (white arrow); : G: On PET-CT, high accumulation is shown in the liver (white arrow) and it matches the MR Image. It is judged as liver metastasis.] This patient has been followed up 72 months and is confirmed to be alive.

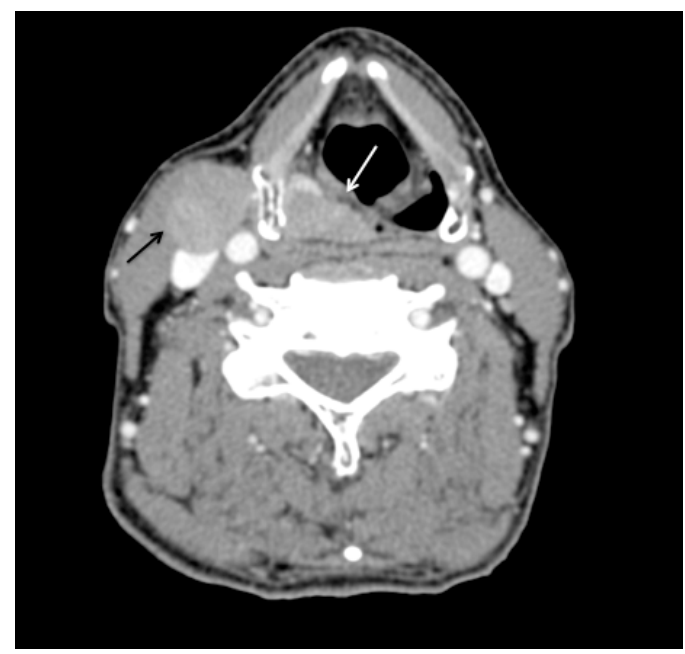

A

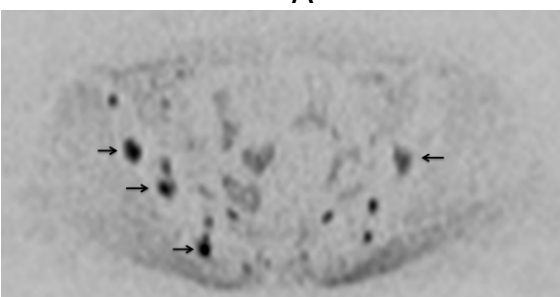

D

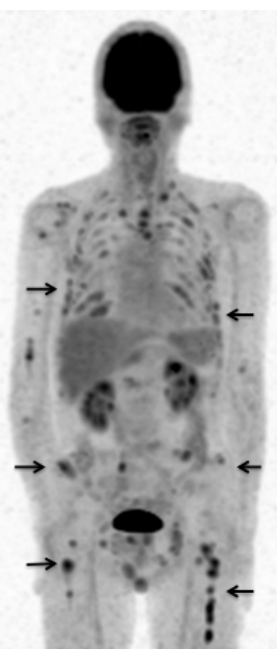

B

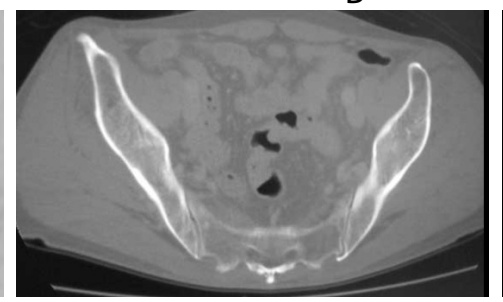

$\mathrm{E}$

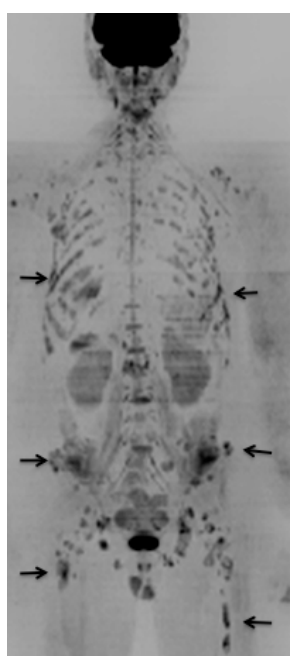

C

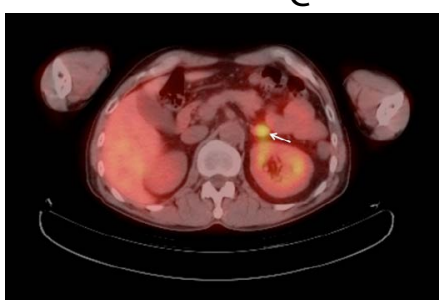

$\mathrm{F}$

Figure 3. A 60-year-old male with bone metastases from HPC (Case 7). The initial stage was T2N2bM0 [A: On initial CT, mass is occupied in the right piriform fossa (white arrow) and lymph node is also shown in the right jugular chain (arrow). SCC was proved by biopsy; B: On MIP image of FDG-PET 7 months after initial CT (Fig.3A), multiple accumulated areas are shown (arrows). These lesions were judged as multiple bone metastases, comprehensively; C: In WB-MRI examination 10 months after initial CT (Fig.3A), in MIP image of DWI, multiple high intensity areas are shown in ribs, bilateral femurs and ilium and so on (arrows). It correlated well with PET-MIP image; D: In original axial image of DWI at the ilium level, multiple high intensity areas of bone metastases are shown (arrows); E: In bone window CT, which is included in PET-CT examination of Fig.3C, on the almost same level of Fig. 3D, neither osteosclerotic nor lytic patterns were shown in the ilium. These lesions were classified as intertrabecular metastases; F: In PET-CT, on the level of adrenal gland, high accumulation is shown in the left adrenal gland and judged as adrenal metastasis (white arrow).] This patient died 20 months after primary site detection. 


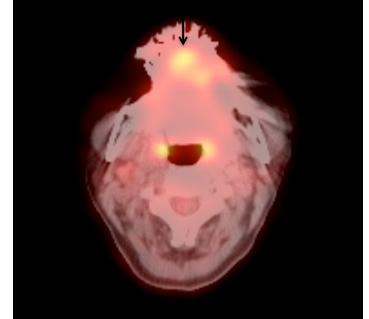

A

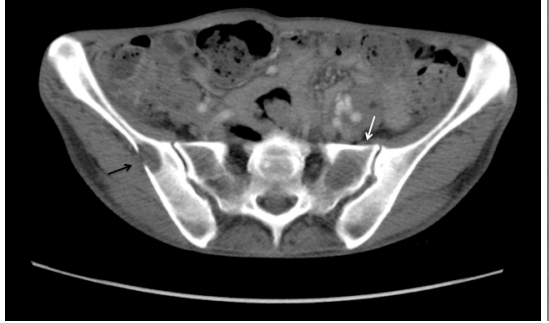

B

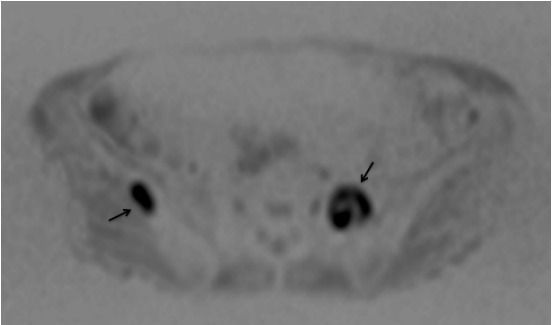

C

Figure 4. A 57-year-old female with bone metastases from tongue carcinoma (case 11). The initial stage was T2N0M0 [A: On initial PET-CT, high accumulation is shown in the middle apex of the tongue (arrow) and SCC was proved by biopsy; B: In CT, after 19 months after initial PET-CT, on the level of pelvis, osteolysis and discontinuity of cortex of the right ilium is shown (arrow) and also osteolysis is shown in the left sacrum (white arrow); C: In DWI of WB-MRI examination 15 days after CT (Figure 4B), high intensity areas are shown in the right ilium and left sacrum (arrows).] These areas were judged as osteolytic metastases comprehensively. In this case, lung and liver metastases appeared at the same time. This patient died 24 months after primary site detection.

Table 1. The list of the cases of bone metastases from HNSCC.

\begin{tabular}{|c|c|c|c|c|c|c|c|c|c|c|c|}
\hline $\begin{array}{l}\text { Case } \\
\text { No. }\end{array}$ & Age/sex & Disease/histology & TNM(initial) & Prev. tr. & $\begin{array}{c}\text { Follow-up } \\
\text { (mth.) }\end{array}$ & $\begin{array}{l}\text { Span } \\
\text { (mth.) }\end{array}$ & $\begin{array}{l}\text { Onset of Metastatic } \\
\text { site } \rightarrow \text { Progression }\end{array}$ & Distribution & $\begin{array}{c}\text { DWI } \\
\text { signal }\end{array}$ & CT findings & Remarks \\
\hline 1 & $54 / \mathrm{M}$ & $\mathrm{NPC} / \mathrm{SCC}$ & T2bN2M0 & CRT & 38 (alive) & 0 & Bone $\rightarrow$ Lung\&Liver & L3 & high & Sclerotic & EBV \\
\hline 2 & $42 / \mathrm{F}$ & $\mathrm{NPC} / \mathrm{SCC}$ & T1N2M0 & CRT & 27 (alive) & 7 & Bone & Multiple & high & Sclerotic & EBV \\
\hline 3 & $53 / \mathrm{M}$ & $\mathrm{OPC} / \mathrm{SCC}$ & T4aN2cM0 & CRT & 72 (alive) & 25 & Bone \&Liver & Multiple & high & Sclerotic & HPV 16 \\
\hline 4 & $60 / \mathrm{M}$ & $\mathrm{OPC} / \mathrm{SCC}$ & T3N2cM0 & CRT & 32 (died) & 11 & Bone \& Liver & Multiple & high & Sclerotic & HPV 16 \\
\hline 5 & $52 / \mathrm{M}$ & OPC/SCC & T2N2bM0 & $\mathrm{RT}$ & 30 (alive) & 12 & Bone & Multiple & high & Sclerotic & HPV 16 \\
\hline 6 & $69 / \mathrm{M}$ & $\mathrm{HPC} / \mathrm{SCC}$ & T4N2bM0 & Op.+RT & 97 (died) & 21 & Bone & Multiple & high & Intertrabecular & \\
\hline 7 & $60 / \mathrm{M}$ & $\mathrm{HPC} / \mathrm{SCC}$ & $\mathrm{T} 2 \mathrm{~N} 2 \mathrm{bM} 0$ & CRT & $20($ died $)$ & 5 & Bone. adr. gld. \& LN & Multiple & high & Intertrabecular & \\
\hline 8 & $55 / \mathrm{M}$ & Tongue/SCC & T1N0M0 & CRT & 18 (died) & 14 & Bone \& Lung & Multiple & high & Intertrabecular & \\
\hline 9 & $43 / \mathrm{M}$ & Tongue/SCC & T1N0M0 & Op. + RT & 14 (died) & 8 & $\mathrm{LN} \rightarrow$ Bone $\rightarrow$ Brain & $\begin{array}{l}\text { Sternum, C6, lt- } \\
\text { humeurs }\end{array}$ & high & Lytic & \\
\hline 10 & $36 / F$ & Tongue/SCC & T1N0M0 & $\mathrm{Op}+\mathrm{RT}$ & 34 (alive) & 24 & LNBone $\rightarrow$ Liver & Multiple & high & Lytic & \\
\hline 11 & $57 / \mathrm{F}$ & Tongue/SCC & T2N0M0 & Op. & 24 (died) & 16 & $\begin{array}{l}\text { Bone, LN, Lung \& } \\
\text { Liver }\end{array}$ & Ilium, Sacrum & high & Lytic & \\
\hline 12 & $66 / \mathrm{M}$ & Larynx/ SCC & $\mathrm{T} 2 \mathrm{~N} 2 \mathrm{bM} 0$ & RT & $25($ died $)$ & 11 & Bone & L5 & high & Lytic & \\
\hline 13 & $58 / \mathrm{M}$ & Larynx/SCC & T3N0M0 & CRT & 6 (died) & 4 & Bone $\rightarrow$ Lung & Multiple & high & Intertrabecular & \\
\hline 14 & $45 / \mathrm{M}$ & Nasal cavity/SCC & T2N0M0 & Op. + RT & 67 (alive) & 42 & LungBone & C6, Sacrum & high & Lytic & \\
\hline
\end{tabular}

NPC: Nasopahryngeal carcinoma; OPC: Orophalyngcal carcinoma; HPC: Hvpopharyngeal carcinoma; Prev. tr.: Previous treatment; CRT: Chemotherapy and radiotherapy; RT: Radiotherapy; Op.: Operation; adr. gld.: Adrenal gland; LN: Lymph node; DWI: Diffusion-weighted images; EBV: Epstein-Barr virus; HPV16: Human pappillorna virus16

Table 2. Protocol of WB-MRI: total spine sagittal sequences.

\begin{tabular}{|l|l|l|}
\hline \multicolumn{1}{|l|}{} & 1.T1WI fast SE & 2. STIR \\
\hline TR/TE/Tl (msec.) & $600 / 9.4$ & $6000 / 80 / 9.4$ \\
\hline ETL & 2 & 14 \\
\hline Slice thickness $(\mathrm{mm})$ & 4 & 4 \\
\hline FOV of each station $(\mathrm{mm})$ & 420 & 420 \\
\hline Matrix & $336 \times 448$ & $250 \times 384$ \\
\hline No. of stations & 2 & 2 \\
\hline Time of each station & 1 min. 14 sec. & 2 min. 2 sec. \\
\hline Other & Fip angle 120 deg. & \\
\hline
\end{tabular}

Table 3. Protocol of WB-MRI: body Coronal sequences.

\begin{tabular}{|l|l|}
\hline TR/TE (msec.) & $120 / 1.2$ (opposed phase), 2.5 (in phase) \\
\hline ETL & 1 \\
\hline Slice thickness $(\mathrm{mm})$ & 7 \\
\hline FOV of each station(mm) & 480 \\
\hline Matrix & $256 \times 179$ \\
\hline No. of stations & 2 with 2 acquisitions \\
\hline Time of each station $(\mathrm{sec})$. & 23 per an acquisition \\
\hline Other & Breath hold scan \\
\hline
\end{tabular}

The numbers of these cases are too few. However, NPC is closely related to Epstein-Barr virus (EBV), peculiar natural history and a good prognosis [11] and OPC is also closely related with Human papilloma virus (HPV) 16 [12]. Indeed, in our two cases of NPC, EBV and also in our three cases of OPC, HPV 16 were proven. Hence, these findings
Table 4. Protocol of WB-MRI: diffusion-weighted axial images.

\begin{tabular}{|l|l|}
\hline TR/TE/Tl (msec.) & $14000 / 60 / 240$ \\
\hline ETL & 1 \\
\hline Slice thickness $(\mathrm{mm})$ & 4 without interslice gap \\
\hline FOV of each station(mm) & 450 \\
\hline Matrix & $79 \times 140$ \\
\hline No. of stations & 4 or 5 \\
\hline Time of each station & 2 min 39 sec. \\
\hline Other & b value 0 and $800 \mathrm{~mm}$ \\
\hline
\end{tabular}

might be the features of SCC of NPC and OPC, compared with the other HNSCC.

We have to pay attention to the four cases of intertrabecular pattern. This pattern is clinically problematic in overall bone metastases, not exclusively in HNSCC $[13,14]$. In this pattern, WB-MRI including DWI, as well as FDG-PET can detect this abnormality but conventional CT or Technecium- $99 \mathrm{~m}$ bone scintigraphy cannot.

\section{Summary}

Bone metastases from HNSCC are rare but need to be detected. It is usually found with lung or liver metastases. However, bone metastasis alone is a possible condition. CT reveals as various patterns and DWI of MRI as high intensity, consistently. In the case of NPC and OPC which are closely related to viral infection, osteosclerotic metastases 
might be characteristic. WB-MRI is a promising tool for detecting bone metastases of HNSCC.

\section{Acknowledgment}

Authors acknowledge the MRI technical staff for cooperating with the rapid and exact image processing of WB-MRI.

\section{References}

1. Kim MR, Roh JL, Kim JS, Choi SH, Nam SY, et al. (2008) 18F-fluorodeoxyglucosepositron emission tomography and bone scintigraphy for detecting bone metastases in patients with malignancies of the upper aerodigestive tract. Oral Oncol 44: 148-152. [Crossref]

2. Noij DP, Boerhout EJ, Pieters-van den Bos IC, Comans EF, Oprea-Lager D, et al. (2014) Whole-body-MR imaging including DWIBS in the work-up of patients with head and neck squamous cell carcinoma: a feasibility study. EJR Eur J Radiol 83: 1144-1151. [Crossref]

3. Peters TT, Senft A, Hoekstra OS, Castelijns JA, Witte BI, et al. (2015) Pretreatment screening on distant metastases and head and neck cancer patients: Validation of risk factors and influence on survival. Oral Oncol 51: 267-271. [Crossref]

4. de Bree R, Haigentz M Jr, Silver CE, Paccagnella D, Hamoir M, et al. (2012) Distant metastases from head and neck squamous cell carcinoma. Part II. Diagnosis. Oral Oncol 48: 780-786. [Crossref]

5. Yi X, Fan M, Liu Y, Zhang H, Liu S (2013) 18 FDG PET and PET-CT for the detection of bone metastases in patients with head and neck cancer. A meta-analysis. $J$ Med Imaging Radiat Oncol 57: 674-679. [Crossref]
6. Buckley JG, Ferlito A, Shaha AR, Rinaldo A (2001) The treatment of distant metastases in head and neck cancer--present and future. ORL J Otorhinolaryngol Relat Spec 63: 259-264. [Crossref]

7. Pearce T, Philip S, Brown J, Koh DM, Burn PR (2012) Bone metastases from prostate, breast and multiple myeloma: differences in lesion conspicuity at short-tau inversion recovery and diffusion-weighted MRI. Br J Radiol 85: 1102-1106. [Crossref]

8. Lecouvet FE, Mouedden J, Collette L, Coche E, Danse E, et al. (2012) Can whole-body magnetic resonance imaging with diffusion-weighted imaging replace Tc $99 \mathrm{~m}$ bone scanning and computed tomography for single-step detection of metastases in patients with high-risk prostate cancer? Eur Urol 62: 68-75. [Crossref]

9. Nakanishi K, Kobayashi M, Nakaguchi K, Kyakuno M, Hashimoto N, et al. (2007) Whole-body MRI for detecting metastatic bone tumor: diagnostic value of diffusionweighted images. Magn Reson Med Sci 6: 147-155. [Crossref]

10. Puri KS, Suresh KR, Gogtay NJ, Thatte UM (2009) Declaration of Helsinki, 2008 implications for stakeholders in research. J Postgrad Med 55: 131-134. [Crossref]

11. Chiesa F, De Paoli F (2001) Distant metastases from nasopharyngeal cancer. ORL $J$ Otorhinolaryngol Relat Spec 63: 214-216. [Crossref]

12. Sanguineti G, Pai S, Agbahiwe H, Ricchetti F, Westra W, et al. (2014) HPV-related oropharyngeal carcinoma with Overt Level two?? metastases at presentation: The risk of subclinical diseases in ipsilateral levels IB. Acta Oncol 53: 662-668. [Crossref]

13. Yamaguchi T, Tamai K, Yamato M, Honma K, Ueda Y, et al. (1996) Intertrabecular pattern of tumors metastatic to bone. Cancer 78: 1388-1394. [Crossref]

14. Yamaguchi T (2001) Intertrabecular vertebral metastases: metastases only detectable on MR imaging. Semin Musculoskelet Radiol 5: 171-175. [Crossref]

Copyright: (C2017 Nakanishi K. This is an open-access article distributed under the terms of the Creative Commons Attribution License, which permits unrestricted use, distribution, and reproduction in any medium, provided the original author and source are credited. 